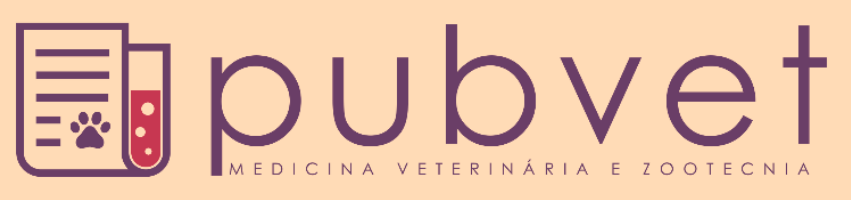

https://doi.org/10.31533/pubvet.v13n01 a258.1-6

\title{
Polirradiculoneurite idiopática aguda em canino soropositivo (IgG) para Toxoplasma gondii
}

\author{
Tainá Ança Evaristo ${ }^{1}$, Beatriz Persici Maroneze ${ }^{2} \bullet$, Bruna dos Santos Pires ${ }^{3}$, Tatiana de
} Ávila Antunes $^{4}{ }^{\ominus}$, Marta Zielke ${ }^{5}$, Liliane Cristina Dias Jerônimo ${ }^{6}{ }^{\bullet}$, Diego Moscarelli

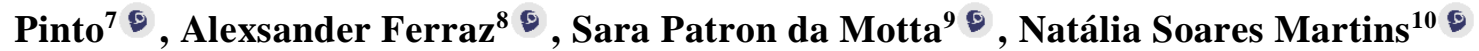

${ }^{I}$ Graduanda em Medicina Veterinária - Universidade Federal de Pelotas (UFPel); Pelotas-RS; Brasil.

${ }^{2}$ Especialista em Clínica Médica Cirúrgica de Animais de Companhia, UFPel Pelotas-RS; Brasil.

${ }^{3}$ Pós-graduanda em Clínica Médica Cirúrgica de Animais de Companhia, UFPel; Pelotas-RS, Brasil.

${ }^{4}$ Pós-graduanda em Residência Multiprofissional em Zoonoses Parasitárias - UFPel, Pelotas-RS, Brasil.

${ }^{5}$ Médica Veterinária Especialista em Clínica de Pequenos Animais, (UFPel); Pelotas-RS; Brasil.

${ }^{6}$ Médica Veterinária, Pelotas-RS, Brasil.

${ }^{7}$ Docente do Departamento de Medicina Veterinária Preventiva, UFPel; Pelotas-RS, Brasil.

${ }^{8}$ Doutorando em Clínica de Pequenos Animais -UFPel; Pelotas-RS, Brasil.

${ }^{9}$ Mestranda em Programa de Pós-graduação em Parasitologia, UFPel; Pelotas-RS, Brasil.

${ }^{10}$ Doutoranda em Programa de Pós-graduação em Parasitologia, UFPel; Pelotas-RS, Brasil.

*Autor para correspondência, E-mail: evaristo.medvet@gmail.com

\begin{abstract}
Resumo. A polirradiculoneurite idiopática aguda canina é uma enfermidade imunomediada e a forma mais comum de polineuropatia aguda em cães. Os sinais clínicos normalmente associados a esta afecção estão relacionados à paralisia flácida dos membros, resultante da sensibilização nos ramos dos nervos espinhais, geralmente não apresentando perda de sensibilidade. Os tratamentos variam de cuidados clínicos e terapêuticos apresentando viabilidade na redução dos sinais clínicos e reabilitação do animal, em grande parte dos casos, de forma completa. O monitoramento do animal é de suma relevância para que a sintomatologia não retorne de forma agressiva, causando maiores prejuízos ao paciente. É necessário o diagnóstico diferencial de intoxicação, botulismo ou Miastenia gravis fulminante e anticorpos de memória para protozoários como Toxoplasma gondii e Neospora caninum. O objetivo do presente trabalho foi relatar a ocorrência de polirradiculoneurite idiopática aguda em canino encaminhado ao Hospital de Clínicas Veterinárias (HCV) na Universidade Federal de Pelotas. Os resultados foram obtidos após diagnóstico clínico e terapêutico, tendo melhora no quadro em aproximadamente seis semanas, sendo este tratamento totalmente sintomático.
\end{abstract}

Palavras chave: cão, neuropatia, tetraplegia flácida simétrica, toxoplasmose.

\section{Acute idiopathic polyradiculoneuritis in seropositive canine (IgG) for Toxoplasma gondii}

\footnotetext{
Abstract. Acute canine idiopathic polyradiculoneuritis is an immune-mediated disease and a more common form of acute polyneuropathy in dogs. Lawyers have been related to the response to limb flaccidity, resulting in the understanding of the nerve and spinal branches, usually without loss of sensitivity. Clinical and therapeutic self-variability are manifested through the clinical response and rehabilitation of the animal, in most cases, completely. The monitoring of the animal is of great relevance for a symptomatology not returned aggressively, causing a great repercussion to the patient. Differential detection, intoxication, toxicity or femoral infection and memory antibodies to protozoa such as Toxoplasma gondii and Neospora caninum are required. The report was submitted to a study of acute idiopathic polyradiculoneuritis in canine sent to the Hospital of Veterinary
} 
Clinics (HCV) of the Federal University of Pelotas. The results were negative to the clinical and therapeutic diagnosis, having obtained an improvement in the quality of life, being this a totally symptomatic treatment.

Keywords: dog, neuropathy, symmetrical flaccid tetraplegia, toxoplasmosis

\title{
Polirradiculoneuritis idiopática aguda en canino seropositivo (IgG) para Toxoplasma gondii
}

\begin{abstract}
Resumen. La polirradiculoneuritis idiopática aguda canina es una enfermedad inmunomediada y una forma más común de polineuropatía aguda en perros. Los signos clínicos normalmente relacionados a esta enfermedad están relacionados con la parálisis flácida de los miembros, resultante de la comprensión de las ramas nerviosas espinales, en general sin pérdida de sensibilidad. Los tratamientos varían de cuidados clínicos y terapéuticos presentando viabilidad en la reducción de los signos clínicos y rehabilitación del animal, en la mayoría de los casos, de forma completa. El monitoreo del animal es de suma relevancia para que la sintomatología no retorne de forma agresiva, causando una gran repercusión al paciente. Es necesario el diagnostico diferencial de intoxicación, botulismo, infección femoral y anticuerpos de memoria para protozoarios como Toxoplasma gondii y Neospora caninum. El objetivo del presente trabajo fue informar el caso de polirradiculoneuritis idiopática aguda en canino encaminado al Hospital de Clínicas Veterinarias (HCV) de la Universidad Federal de Pelotas. Los resultados fueron obtenidos después del diagnóstico clínico e terapéutico, habiendo obtenido una mejora en la calidad de vida en seis semana de tratamiento, siendo éste un tratamiento totalmente sintomático.
\end{abstract}

Palabras clave: perro, neuropatía, tetraplejia flácida simétrica, toxoplasmosis

\section{Introdução}

A Polirradiculoneurite Idiopática Aguda Canina (PIAC) trata-se de uma patologia relativamente comum, gerada pela inflamação nos ramos ventrais dos nervos espinhais, podendo progredir de forma caudo-cranial, dependendo do agravamento do quadro, aos ramos dorsais (Añor, 2014; Dewey, 2006). É considerada equivalente a neuropatia aguda em humanos intitulada Síndrome de Guillan-Barré (SGB) (Cuddon, 2002). Tendo uma distribuição ampla, pode atingir cães de várias raças e idades, de ambos os sexos (Slatter, 2007). Como a síndrome trata-se de um quadro progressivo caudo-cranial de paralisia músculo-esquelética, quando não tratado, pode ocorrer paralisia dos músculos responsáveis pela respiração, levando o paciente a óbito (Nelson \& Couto, 2015).

As lesões em neurônio motor inferior (NMI) são consequência de dano que envolve o corno ventral espinhal e/ou o próprio NMI em alguma de suas porções, como corpo celular, axónio, placa motora ou músculo (Uemura, 2015). Animais com tetraparesia aguda de NMI habitualmente apresentam disfunção motora dos membros, pescoço e, por vezes, paresia ou paralisia respiratória, bem como alterações significativas nos nervos cranianos causando, por exemplo, dificuldade de deglutição e vocalização (Añor, 2014; Bojrab, 2005).

A PIAC foi descrita pela primeira vez em 1954 por Kingma \& Catcott (1954), como uma paralisia flácida ascendente que se desenvolvia em cães sete até dez dias após terem sido expostos a saliva de guaxinins através de mordedura, sendo designada como Paralisia dos cães da raça Coonhound (PCH). Atualmente, considera-se a PIAC uma doença imunomediada que afeta os axônios, a bainha de mielina neural ou ambos, nas raízes nervosas ventrais dos nervos espinhais, geralmente com quadro agudo de paraparesia de NMI, que progride para tetraparesia ou tetraplegia, acompanhada de disfunção dos nervos cranianos (Cuddon, 2002; Dewey, 2006). Todavia, independente da origem, os sinais clínicos são idênticos em todos os casos descritos (Añor, 2014). Portanto, o termo PCH refere-se a cães com história antecedente de mordidas ou arranhões de guaxinis, enquanto cães com o mesmo sinal clínico, mas sem exposição à saliva, designa-se de PIAC (Cuddon, 2002; Dewey, 2006). Segundo Vaquero (2015) estas duas subcategorias provavelmente referem-se à mesma doença, mas com fatores de predisposição desconhecidos ou, na maioria dos casos, incomprovado o seu papel de desenvolvimento. Por fim, a 
PIAC associada a protozoários está descrita em cães jovem, principalmente causada por Toxoplasma gondii e Neospora caninum, sendo considerada a classe IgG positiva para ambos parasitos fator de prédisposição para síndrome (Vaquero, 2015).

O objetivo do presente trabalho é relatar a ocorrência de um caso de Polirradiculoneurite Idiopática Aguda em um canino, IgG positivo para Toxoplasma gondii, encaminhado para atendimento no Hospital de Clínicas Veterinárias (HCV) na Universidade Federal de Pelotas (UFPel).

\section{Material e métodos}

Foi encaminhado para o Hospital de Clínicas Veterinária (HCV), na Universidade Federal de Pelotas (UFPel), um canino, SRD, idoso, pesando $35 \mathrm{~kg}$, com histórico de possível quadro de convulsão e disfunção da musculatura dos membros pélvicos, com flacidez muscular.

No exame clínico geral, o animal apresentava paralisia de membros pélvicos, com nível de consciência progressivamente melhorando, apresentando-se em estupor. A temperatura retal apresentava em $38,2^{\circ} \mathrm{C}$, as mucosas manifestavam-se coloração hiperêmica, com hidratação não aparente $(<5 \%)$. O paciente apresentava comportamento calmo, sendo internado para melhor averiguação do quadro. Os parâmetros do cão que foram avaliados na Unidade de Internação Veterinária (UIV) estão expressos na Tabela 1.

Tabela 1. Ficha de monitoração de um canino após encaminhamento para Unidade de Internação Veterinária, localizado no Hospital de Clínicas Veterinárias, no setor de Clínica de Pequenos Animais, Universidade Federal de Pelotas (UFPel)

\begin{tabular}{lcccc}
\hline & \multicolumn{4}{c}{ Ficha de Monitoração - Unidade de Internação Veterinária } \\
\hline Parâmetros & $14: 00$ & $14: 30$ & $15: 00$ & $15: 30$ \\
FC (BPM) & 90 & 84 & 84 & 92 \\
FR (MRPM) & 16 & 16 & 16 & 12 \\
$\Delta \mathrm{T}\left(\mathrm{C}^{\circ}\right)$ & 37,1 & 37,1 & 37 & 36,9 \\
TPC $(\mathrm{s})$ & 3 & 3 & 2 & 2 \\
PM & Hiperêmica & Hiperêmica & Hiperêmica & Hiperêmica
\end{tabular}

Nota: FC - frequência cardíaca em batimentos por minuto (BPM); FR - frequência respiratória em movimentos respiratórios por minuto (MRPM); $\Delta \mathrm{T}$ - variação de temperatura em graus centígrados $\left(\mathrm{C}^{\circ}\right)$; TPC - tempo de perfusão capilar em segundos (s'); PM - pigmentação de mucosas.

O paciente foi encaminhado para realização de exames complementares laboratoriais e de imagem. Os resultados do hemograma e bioquímico estavam dentro dos padrões fisiológicos adotados para a espécie; porém, houve constatação de acidez discreta no $\mathrm{pH}$ urinário $(7,5)$ e presença de proteínas. Além disso, foi solicitado a realização de radiografia torácica (Figura 1), sendo constatada leve alteração na silhueta cardíaca.

No diagnóstico presuntivo suspeitava-se de Acidente Vascular Cerebral (AVC), botulismo ou intoxicação. Após exame neurológico, o diagnóstico definitivo foi classificado como Polirradiculoneurite idiopática canina aguda, conforme os sinais clínicos demonstrados pelo paciente. Conforme o diagnóstico solicitou-se a internação do paciente no HCV. Como terapia inicial de suporte, utilizou-se fluidoterapia com ringer com lactato de sódio ( $10 \mathrm{ml} / \mathrm{kg}$, I.V.); furosemida ( $2 \mathrm{mg} / \mathrm{kg}$, I.V.); omeprazol (2,2 ml, I.V. 12 em 12 horas) por dez dias.

Durante os primeiros dias de internação houve uma progressão no quadro neuropático, o paciente demonstrou grande dificuldade para locomover-se, maior flacidez muscular nos membros torácicos e não se alimentava. Foi necessário utilizar uma sonda esofágica nos primeiros dias de terapia, havendo fornecimento de alimentação pastosa hipercalórica por sete dias. Durante o período de internação, houve redução considerável do peso corporal do paciente, reduzindo de $35 \mathrm{~kg}$ para $26 \mathrm{~kg}$. Além disso, foi utilizada a sondagem uretral por sete dias, visto a dificuldade de locomoção do canino para efetuar a adequada micção.

Sendo de suma importância para progressão e reabilitação do paciente, o tratamento realizado foi feito não apenas com fármacos, mas também realizando monitoramento do bem-estar do paciente, com 
troca de seis em seis horas de decúbito, já que a paralisia flácida dos membros pélvicos impossibilitava a locomoção do canino.

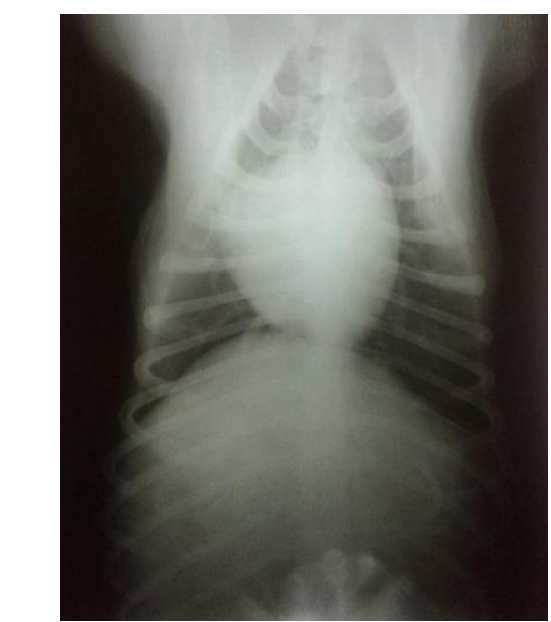

Figura 1. Radiografia torácica de canino com discreto aumento na silhueta cardíaca.

Conforme o caso, foi solicitado o exame de Imunofluorescência Indireta (RIFI) para anticorpos da classe IgG para Toxoplasma gondii e Neospora caninum. O paciente apresentou anticorpos de memória apenas para $T$. gondii. Após este resultado, iniciou-se o tratamento com Clindamicina via oral por 30 dias $(20 \mathrm{mg} / \mathrm{kg} /$ B.I.D).

\section{Resultados e discussão}

Após 45 dias de tratamento e internação, o paciente apresentou reversão total dos sinais clínicos referentes à PIAC, tendo alta clínica. Neste período o canino melhorou seu escore corporal, havendo ganho de peso de $26 \mathrm{~kg}$ para $30 \mathrm{~kg}$.

Quanto a PIAC associada a protozoários, os parasitos mais comumente documentados são Toxoplasma gondii e Neospora caninum (Vaquero, 2015), o que vem de acordo com o presente relato. Todavia, existem referidos casos de cães com miopatias inflamatórias associadas a Leishmania infantum, Erlichia canis e Hepatozoon canis, que desenvolvem quadro de fraqueza e atrofia muscular generalizada (Paciello et al., 2009; Vaquero, 2015).

Conforme Deplazes (2010) e Dubey \& Lappin (2013), os animais com PIAC associada a protozoários tipicamente desenvolvem paraparesia aguda com extensão rígida dos membros pélvicos, com reflexos patelar e flexor diminuídos com ausência de estímulo em membro pélvico, com contraturas musculares; porém, mantêm-se nociceptivos, o que vem de acordo com os sinais clínicos do relato neste canino. Alguns animais têm progressão da doença de forma fulminante, com desenvolvimento de tetraparesia ou tetraplegia com insuficiência respiratória, porém não houve paralisia respiratória neste caso.

O paciente apresentava-se em estupor ao ser avaliado no exame clínico pré-internação, o que vem de acordo ao que cita Dewey (2006), sendo importante observar e fazer a avaliação do estado de consciência do animal, bem como a capacidade de interação ao ambiente.

Um estudo recente realizado em cães tentou estabelecer uma relação entre PIAC e evidência sorológica a exposição de Erlichia canis, Borrelia bugdorferi, Toxoplasma gondii, Neospora caninum, Campylobacter jejuni e o vírus da esgana, concluindo que a infecção por toxoplasmose pode ser fator de desencadeamento da doença (Holt et al., 2011). Trata-se de suma relevância o diagnóstico diferencial de PIAC de outras três maiores causas de tetraparesia/tetraplegia aguda progressiva de NMI: o botulismo, a paralisia da Carraça e Miastenia Gravis aguda fulminante (Añor, 2014; Cuddon, 2002; Vaquero, 2015). Além de outras causas menos comuns que englobam doenças como a desernervação distal, a neuropatia retardada induzida por intoxicação a organofosforados, envenenamento por cobracoral, intoxicação por alga-verde, envenenamento causado por aranha viúva-negra (fase final do envenenamento) e outras intoxicações raras, como por exemplo, por lasalocida (agente antibacteriano e coccidiostático) (Añor, 2014; Espino et al., 2003; Griffiths \& Duncan, 1979; Hopper et al., 2002; 
Karalliedde et al., 2006; Pérez et al., 2012; Peterson, 2006; Pittman et al., 2012; Segev et al., 2004; Vaquero, 2015).

Quanto às doenças que causam um quadro clínico de tetraparesia de NMI de forma crônica progressiva, cita-se as endocrinopatias (Diabetes mellitus, hipotireoidismo e hiperadrenocorticismo, síndromes paraneoplásicas (insulinoma, linfossarcoma, adenocarcinomas, carcinomas e sarcomas), toxinas, fármacos (cisplastina, vincristina e vinblastina), infeções (por N. caninum ou por T. gondii), doenças imunomediadas (Lúpus eritematoso sistémico) e causas idiopáticas (degeneração axonal crónica) (Añor, 2014; Cuddon, 2002; Troxel, 2007).

Conforme a anamnese, exame clínico geral, exame clínico específico neurológico, exames complementares laboratoriais e de imagem, houve a exclusão de todas as possíveis causas de tetraparesia/tetraplegia aguda progressiva caudo-cranial de NMI. Além disso, a presença de anticorpos de memória ( $\mathrm{IgG}$ ) para Toxoplasma gondii é um fator de predisposição para a ocorrência da polirradiculoneurite aguda, o que vem de acordo com a literatura (Añor, 2014; Cuddon, 2002; Vaquero, 2015).

Após a fase progressiva da doença os sinais clínicos estabilizam e a maior parte dos animais recuperase entre três e seis semanas, o que vem de acordo ao caso, pois o paciente apresentou regressão completa destes em aproximadamente seis semanas (Hirschvogel et al., 2012).

\section{Conclusão}

O paciente recuperou-se em seis semanas, concluindo o tratamento terapêutico para Toxoplasma gondii, sem apresentar nenhuma sintomatologia clínica referente a polirradiculoneurite idiopática aguda canina, tendo alta clínica. Desta forma, demonstra-se de suma relevância a exclusão de possíveis causas clínicas para síndromes neurológicas e a utilização de exames complementares para chegar ao diagnóstico final neste caso.

\section{Referências bibliográficas}

Añor, S. (2014). Acute lower motor neuron tetraparesis. Veterinary Clinics: Small Animal Practice, 44(6), 1201-1222.

Bojrab, M. J. (2005). Técnicas atuais em cirurgia de pequenos animais. São Paulo: Editora Roca.

Cuddon, P. A. (2002). Acquired canine peripheral neuropathies. Veterinary Clinics: Small Animal Practice, 32(1), 207-249.

Deplazes, P. (2010). Parasitic neurological diseases of the dog and cat. In A. Jaggy \& S. Platt (Eds.), Atlas and textbook of small animal neurology: An illustrated text. Schluetersche, Germany.

Dewey, C. W. (2006). Encefalopatias: distúrbios cerebrais (Vol. 1). São Paulo: Roca.

Dubey, J. P. \& Lappin, M. R. (2013). Toxoplasmosis and neosporosis. In C. E. Greene (Ed.), Infectious diseases of the dog and cat. Amsterdan, Holanda: Elsevier Health Sciences.

Espino, L., Suarez, M. L., Miño, N., Goicoa, A., Fidalgo, L. E. \& Santamarina, G. (2003). Suspected lasalocid poisoning in three dogs. Veterinary and Human Toxicology, 45(5), 241-242.

Griffiths, I. R. \& Duncan, I. (1979). Distal denervating disease: a degenerative neuropathy of the distal motor axon in dogs. Journal of Small Animal Practice, 20(10), 579-592.

Hirschvogel, K., Jurina, K., Steinberg, T. A., Matiasek, L. A., Matiasek, K., Beltrán, E. \& Fischer, A. (2012). Clinical course of acute canine polyradiculoneuritis following treatment with human IV immunoglobulin. Journal of the American Animal Hospital Association, 48(5), 299-309.

Holt, N., Murray, M., Cuddon, P. A. \& Lappin, M. R. (2011). Seroprevalence of various infectious agents in dogs with suspected acute canine polyradiculoneuritis. Journal of Veterinary Internal Medicine, 25(2), 261-266.

Hopper, K., Aldrich, J. \& Haskins, S. C. (2002). The recognition and treatment of the intermediate syndrome of organophosphate poisoning in a dog. Journal of Veterinary Emergency and Critical Care, 12(2), 99-103. 
Karalliedde, L., Baker, D. \& Marrs, T. C. (2006). Organophosphate-induced intermediate syndrome. Toxicological Reviews, 25(1), 1-14.

Kingma, F. J. \& Catcott, E. J. (1954). A paralytic syndrome in coonhounds. Veterinary Neurology American, 35, 115-117.

Nelson, R. W. \& Couto, C. G. (2015). Medicina interna de pequenos animais. Amsterdan: Elsevier Editora.

Paciello, O., Oliva, G., Gradoni, L., Manna, L., Manzillo, V. F., Wojcik, S., . . Papparella, S. (2009). Canine inflammatory myopathy associated with Leishmania Infantum infection. Neuromuscular Disorders, 19(2), 124-130.

Pérez, M. L., Fox, K. \& Schaer, M. (2012). A retrospective evaluation of coral snake envenomation in dogs and cats: 20 cases (1996-2011). Journal of Veterinary Emergency and Critical Care, 22(6), 682-689.

Peterson, M. E. (2006). Black widow spider envenomation. Clinical Techniques in Small Animal Practice, 21(4), 187-190.

Pittman, J., Brainard, B. \& Swindells, K. (2012). Neurological toxicities. In S. Platt \& L. Garosi (Eds.), Small animal neurological emergencies: CRC Press.

Segev, G., Baneth, G., Levitin, B., Aroch, I. \& Shlosberg, I. (2004). Accidental poisoning of 17 dogs with lasalocid. Veterinary Record, 155(6), 174-176.

Slatter, D. H. (2007). Manual de cirurgia de pequenos animais. São Paulo: Manole.

Troxel, M. T. (2007). Rapidly progressive lower motor neuron diseases in dogs. Massachusetts Veterinary Referral Hospital, 8(3), 1-10.

Uemura, E. E. (2015). Fundamentals of canine neuroanatomy and neurophysiology. USA: John Wiley \& Sons.

Vaquero, P. M. (2015). Coonhound paralysis (Acute Polyradiculoneuritis). In L. P. Tilley, L. P. Tilley \& F. W. K. Smith (Eds.), Blackwell's five-minute veterinary consult: Canine and feline: Wiley.

Recebido: 17 novembro, 2018

Aprovado: 5 dezembro, 2018.

Publicado: 29 janeiro, 2019.

Licenciamento: Este artigo é publicado na modalidade Acesso Aberto sob a licença Creative Commons Atribuição 4.0 (CC-BY 4.0), a qual permite uso irrestrito, distribuição, reprodução em qualquer meio, desde que o autor e a fonte sejam devidamente creditados 